\title{
System of Modeling and Visualization of Domain of the Heart Excitation
}

\author{
Dmitry Belov \\ Grodno State University, 22, Ozheshko Street, 230023 Grodno, Belarus \\ belov@grsu.grodno.by
}

\begin{abstract}
The mathematical aspects of the problem of the reconstruction of domain of the heart excitation from the body surface potentials are investigated. We propose modeling algorithms allowing to reduce endless set of solutions of the problem. These algorithms take into account local and global time restrictions of the function whose support is equal to the domain. As a result a new model of cardiogenerator is developed. This model operates within the framework of classical model of excitation spreading as a multidipole generator. At the same time our generator provides stable solution of the problem in contrast to multidipole one. In final the system of modeling and visualization of domain of the heart excitation is developed. The clinical tests have shown efficiency of applying of the system for the myocardial infarction diagnostics.
\end{abstract}

\section{Introduction}

The problem of identification of cardiogenerator characteristics from the potentials given on the body surface is called the inverse problem of the electrocardiography. For example, in [7] the problem of identification of characteristics of the potential field on the ventricular surface is investigated. In [3] the problem of identification of electrostatics volume sources inside heart is researched. Let us consider the problem of construction of domain of the heart excitation from the body surface potentials. This problem will be called $\Omega$-problem. From the mathematical point of view the domain of the heart excitation is a support of volume function of the electrostatics sources inside heart, i.e. domain of nonzero values of the function. The fundamental meaning of the problem has been emphasized in [5]. Observing in any time the domain of the heart excitation cardiologist can diagnose various cardiac irregularities. It is known [4], that the problem of determination of the volume function of the electrostatics sources from the surface potentials has non-unique solution. Therefore $\Omega$-problem has non-unique solution as well. Thus the important problem of restriction of the set of solutions of $\Omega$-problem arises.

Going over to the general mathematical aspects of $\Omega$-problem we consider the operator equation of first kind:

$$
A(\boldsymbol{r}, \boldsymbol{s}, t) p(\boldsymbol{r}, t)=u_{\delta}(\boldsymbol{s}, t)
$$


where $t \in[0, T]$ is time, $p(\boldsymbol{r}, t)$ is unknown volume function of electrostatics sources with domain of definition $P(t), A(\boldsymbol{r}, \boldsymbol{s}, t)$ is unknown and non-linear operator, $u_{\delta}(\boldsymbol{s}, t)$ is potentials given on the body surface $U(t)$ with error $\delta>0$. Support $P^{\prime}(t)$ of the $p(\boldsymbol{r}, t)$ is unknown but for any time $P^{\prime}(t) \subset P(t)$ and

$$
\int_{P^{\prime}(t)} p(\boldsymbol{r}, t) d r=\int_{P(t)} p(\boldsymbol{r}, t) d r=0
$$

We suppose functions $p(\boldsymbol{r}, t)$ and $u(\boldsymbol{s}, t)$ belong to the Hilbert spaces $H_{p}$ and $H_{u}=L_{2}(U(t))$ respectively. Thus $\Omega$-problem is a problem of determination of $P^{\prime}(t)$. Obviously we can solve this problem after the construction of the solution of the equation (1). In electrocardiography any solution of (1) is called equivalent cardiogenerator, because it generates the same body surface potentials as desired one. It is wide known multipole [2,3] and multidipole [2,3] equivalent generators. Let us suppose that the body is infinitely wide homogeneous medium, then equation (1) transforms to the equation:

$$
\frac{1}{4 \pi \sigma} \int_{P(t)} \frac{p(\boldsymbol{r}, t)}{\|\boldsymbol{r}-\boldsymbol{s}\|_{R_{3}}} d v=u(\boldsymbol{s}, t)
$$

where $\sigma$ is a specific conductivity of the medium.

For the reconstruction of $P^{\prime}(t)$ we will reject from $P(t)$ points such that $\left|p_{\Omega}(\boldsymbol{r}, t)\right| \leq \tau$, where $\tau$ is a threshold and $p_{\Omega}(\boldsymbol{r}, t)$ is a solution of regularized according to Tikhonov [6] equation (3) solved by Voevodin method [6]. The result subset of $P(t)$ will approximate $P^{\prime}(t)$.

The Tikhonov regularization method is based on the fundamental idea of the restriction of the domain of definition of the integral operator in (3), i.e. restriction of the solutions set of (3). Moreover in our case this restriction is determined by definition of the Hilbert space of function $p(\boldsymbol{r}, t)$. So taking into account biophysics phenomena of heart work we construct Hilbert space of function $p(\boldsymbol{r}, t)$ in which all functions have equal support, and we can identify unique solution of $\Omega$-problem. Thus if equation (3) has non-unique solution then $\Omega$-problem can have unique solution. This fact is very important for electrocardiography applications.

\section{Methods of the Restriction of Solutions Set of $\Omega$-Problem}

Let for given $t$ the following restriction is true:

$$
P(t)=\bigcup_{i=1}^{k} P_{i}(t), \bigcap_{i=1}^{k} P_{i}(t)=\emptyset, k \geq 1, \forall i: \int_{P_{i}(t)} p(\boldsymbol{r}, t) d r=0
$$

It is easy to prove, that functional space $W_{2+}^{1}(P(t))$ with the norm 


$$
\|f(\boldsymbol{r})\|=\sqrt{\int_{P(t)}\left(f^{2}(\boldsymbol{r})+f^{\prime 2}(\boldsymbol{r})\right) d r+\frac{1}{\varepsilon} \sum_{i=1}^{k}\left(\int_{P_{i}(t)} f(\boldsymbol{r}) d r\right)^{2}}
$$

is Hilbert space [1], where $f(\boldsymbol{r}) \in W_{2+}^{1}(P(t))$ and $f^{\prime}(\boldsymbol{r})$ is generalized derivative of first order [1]. It follows, that if $p(\boldsymbol{r}, t)$ belongs to $W_{2+}^{1}(P(t))$ then the method of construction of $P^{\prime}(t)$ stated above is true and solving $\Omega$-problem we take into account restriction (4). In the norm (5) the last member is a penalty.

In computer experiments we reconstruct positions $(\Omega$-problem) and tracks ( $\Omega^{\prime}$-problem) of two moving charges having equal values and different signs from the potentials given on a containing circle, where $\delta=1.0 e-4$. Obviously in this case corresponding equation (3) has non-unique solution. On the fig.1,2 dots correspond to the knots of grid belonging to $P(t)$, squares correspond to solutions of the $\Omega^{\prime}$-problem, circles correspond to current positions of the charges.
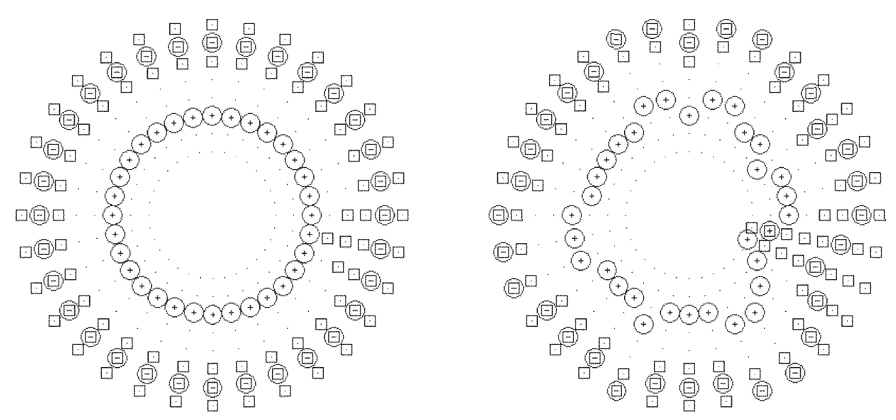

Fig.1. Two solutions of $\Omega^{\prime}$-problem. We have assumed that $p(\boldsymbol{r}, t) \in W_{2}^{1}(P(t))$.
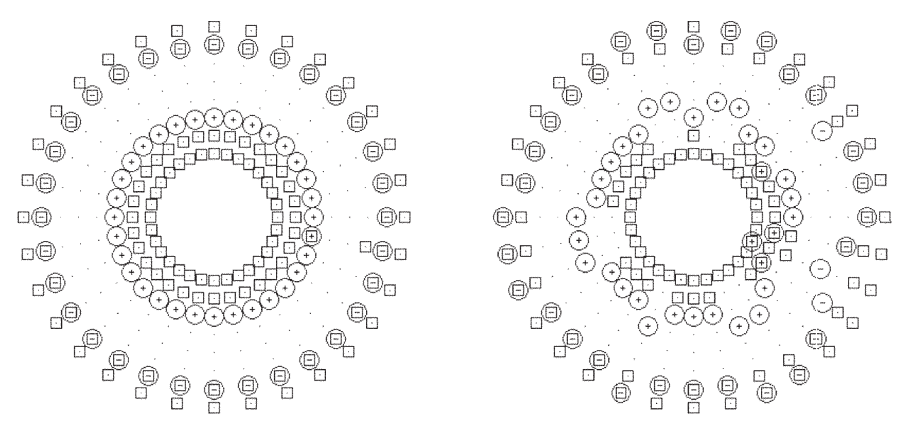

Fig.2. Two solutions of $\Omega^{\prime}$-problem. We have assumed that $p(\boldsymbol{r}, t) \in W_{2+}^{1}(P(t))$.

One can see on fig.1,2 that use of constructed space $W_{2+}^{1}(P(t))$ allows to reconstruct track of positive charge in contrast to use of Sobolev space $W_{2}^{1}(P(t))[1]$. In [9] the results of computer experiments on reconstruction of positions and tracks of dipole on the plain are presented. 
Let for the time segment $[0, T]$ the following condition is true:

$$
G \supset G^{\prime}=\bigcup_{t=0}^{T} P^{\prime}(t), \forall \boldsymbol{r} \in G^{\prime} \int_{0}^{T} p^{2}(\boldsymbol{r}, t) d t=\int_{t(\boldsymbol{r})}^{t(\boldsymbol{r})+\Delta t} p^{2}(\boldsymbol{r}, t) d t,
$$

where $G$ and $G^{\prime}$ are connected sets, and $P^{\prime}(0)$ and $G$ are known. Obviously the condition specifies $3 \mathrm{D}$ object that are continuously changing its form and position in the course of time. For the construction a sequence of $\left\{P\left(t_{i}\right)\right\}$ taking into account (6) we propose the following algorithm:

1. $P\left(t_{0}\right)=P^{\prime}(0), G_{d}=\emptyset$

2. Let $P\left(t_{i}\right)$ is known, then if $M^{\alpha}\left[p, P\left(t_{i}\right)\right] \leq \tau_{s}$ then $P\left(t_{i+1}\right)=P\left(t_{i}\right)$, $G_{d}=G_{d} \cup P\left(t_{i}\right)$. Otherwise, $P\left(t_{i+1}\right)=P_{\Omega}\left(t_{i-1}\right)$ and while for $P\left(t_{i+1}\right)$ inequality $M^{\alpha}\left[p, P\left(t_{i+1}\right)\right]>\tau_{s}$ will be valid we add to $P\left(t_{i+1}\right)$ points from $G$, that are neighbors of points from $P\left(t_{i+1}\right)$ and do not belong to $G_{d} / P_{\Omega}\left(t_{i-1}\right)$. Here $M^{\alpha}\left[p, P\left(t_{i}\right)\right]$ is regularization functional [6] and $\tau_{s}$ is a threshold.

Choosing neighbor points randomly we get algorithm of random search of $\left\{P\left(t_{i}\right)\right\}$.

\section{The Structure of the Equivalent Generator of the Heart}

Solving electrocardiography $\Omega$-problem we have:

1. $P^{\prime}(t)$ is domain of the heart excitation, i.e. support of $p(\boldsymbol{r}, t)$.

2. $U(t)$ is a geometric model of the body surface.

3. $P_{\Omega}(t)$ is model of domain of the heart excitation for the given $t$, i.e. solution of $\Omega$-problem.

4. $P(t)$ is a set containing domain of the heart excitation for given $t$.

5. In (4) each $P_{i}(t)$ is preferable direction of the excitation for the given $t \in[0, T]$. Obviously if restriction (4) is true then restriction (2) is true as well. Is follows restriction (4) specifies the state of classical excitation model for given $t$. Today it is known preferable direction of the excitation for the main parts of the heart [2].

6. In (6) $G$ is a geometric model of the heart, $P^{\prime}(0)$ is a geometric model of sinus knot. Thus restrictions (4) and (6) specify classical model of excitation spreading inside heart.

7. Time segment $[0, T]$ is excitation period.

Thus we developed equivalent generator of the heart, that operates within the framework of classical model of excitation spreading as a multidipole generator. At the same time using regularization technics our generator provides stable solution of the $\Omega$-problem in contrast to multidipole one.

For the development of system of modeling and visualization of domain of the heart excitation we assume:

1. Body surface model $U(t)$ is time fixed truncated cylinder $U$ (fig.3). This analytical model permits to use surface integral in regularization functional [6] and therefore reduces time and space of computations. 


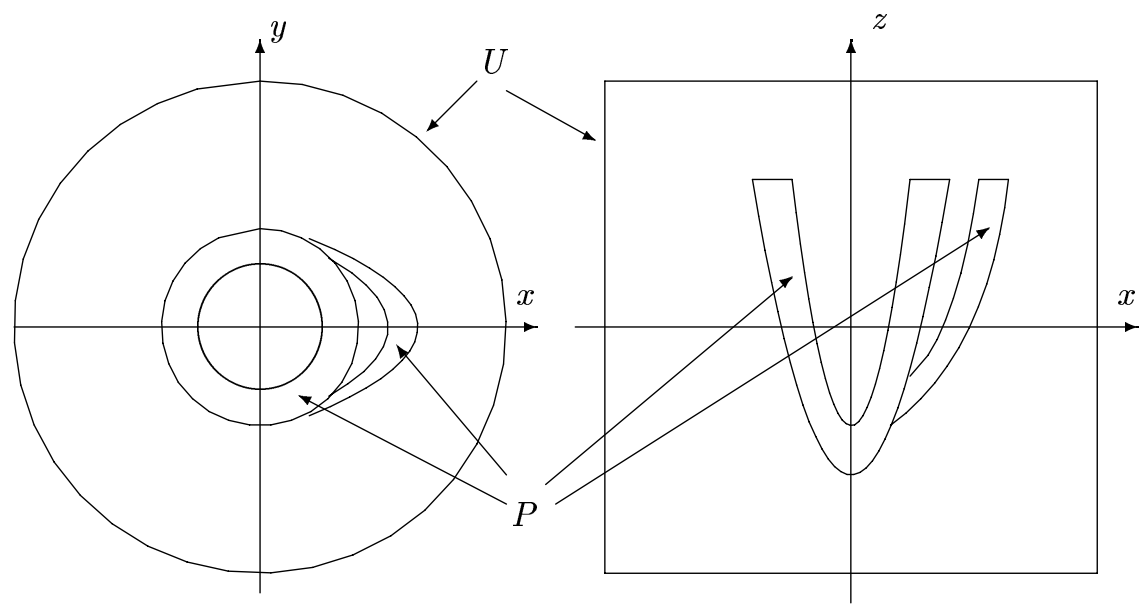

Fig.3. Geometric model of heart and body surface.

2. Heart model $P(t)=G$ is time fixed logical combination $P$ of ellipsoids (fig.3).

3. For the approximation of function $u_{\delta}(s, t)$ on $U$ vectorcardiographic (VCG) data is used.

4. In (4) we assume that $k=1$, i.e. we take into account restriction (2).

5. We do not take into account restriction (6).

\section{System of Modeling and Visualization of Domain of the Heart Excitation}

Let us consider main stages of the system operation:

Preparation: It is regularization of equation (3) and preparation by Voevodin scheme. The results of the stage is saved in the preparation bank. This stage take into account $H_{u}, H_{p}, U$ and $P$.

Solution: Using data from the preparation bank the system numerically solves the equation (3) for the different value of potentials. The solution is resulted on 3D grid and further is saved in solutions bank.

Construction and visualization of the layer-model: Layer-model of 3D object given on $3 \mathrm{D}$ grid is effective alternative for the voxel model, where one needs to identify form and position of the objects only. Geometric properties of the layer-model allow to develop fast visualization algorithms [10]. Let us present our investigation object as a set of generalized vertical cylinders standing bottom on top. Each cylinder, corresponding to the determined contour of the object in layer is approximated with the simple polyhedron. These polyhedrons will be called layers. A set of such layers will be called a layer-model. This approach is considered for computer tomography applications in [8], but for visualization 
authors suggested scan-line algorithm. Obviously it is sufficient to know only the respective polygon and the height of the respective layer for the description of a polyhedron. Let $L$ be a number of layers. Further, if an object of the given set of objects has a maximum number of vertices, then it will be called a maximum object. Let $N$ be a number of vertices of the maximum polygon, then the $O(L N)$ space is sufficient for the presentation of the layer-model. It is easy to prove that elimination of hidden-surface for layer-model runs in optimal $O(\log L+L N \log N)$ time.

Let us consider general sequence of the system operation:

Preparation of the equation $(3) \Rightarrow$ Preparation data bank $\Rightarrow$ Obtaining of the vectorcardiographic (VCG) data $\Rightarrow$ Approximation body surface potentials by $\mathrm{VCG} \Rightarrow$ Solution of the equation $(3) \Rightarrow$ Construction of the layer-model $\Rightarrow$ Visualization of the layer-model.

\section{Clinical Experiments}

System of modeling and visualization of domain of the heart excitation is being used in cardiology department of Grodno region clinical hospital. The system provides $2 \mathrm{D}$ and $3 \mathrm{D}$ visualization of the excitation process in ventricle of a heart.

On fig. 4 the model of domain of the heart excitation of healthy man for the time of the ventricle excitation is presented. On fig.5,6 for the same time the models of domains of the heart excitation of myocardial infarction patients are shown. One can see that for the same threshold value volume of the excitation domain on fig.5,6 is less than one on fig.4. This fact says about partial affection of the conductor cells for sick men. Different configuration of the domains on fig.5,6 says about affection in different parts of the ventricles.

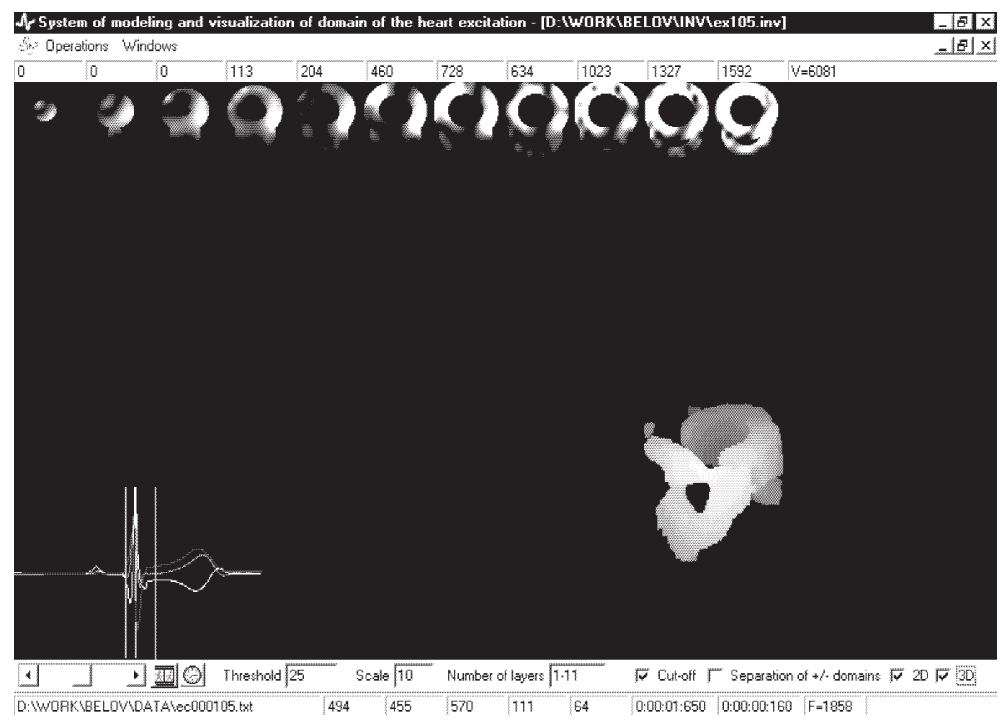

Fig.4. Excitation domain in ventricles zone for the healthy man. 


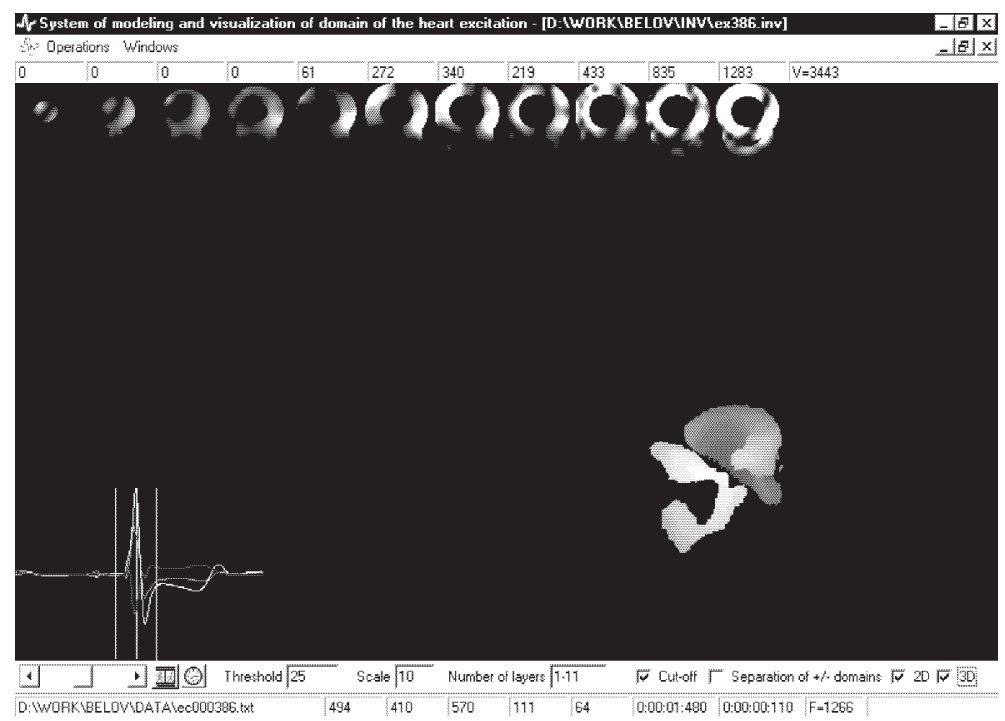

Fig.5. Excitation domain in ventricles zone for the sick man.

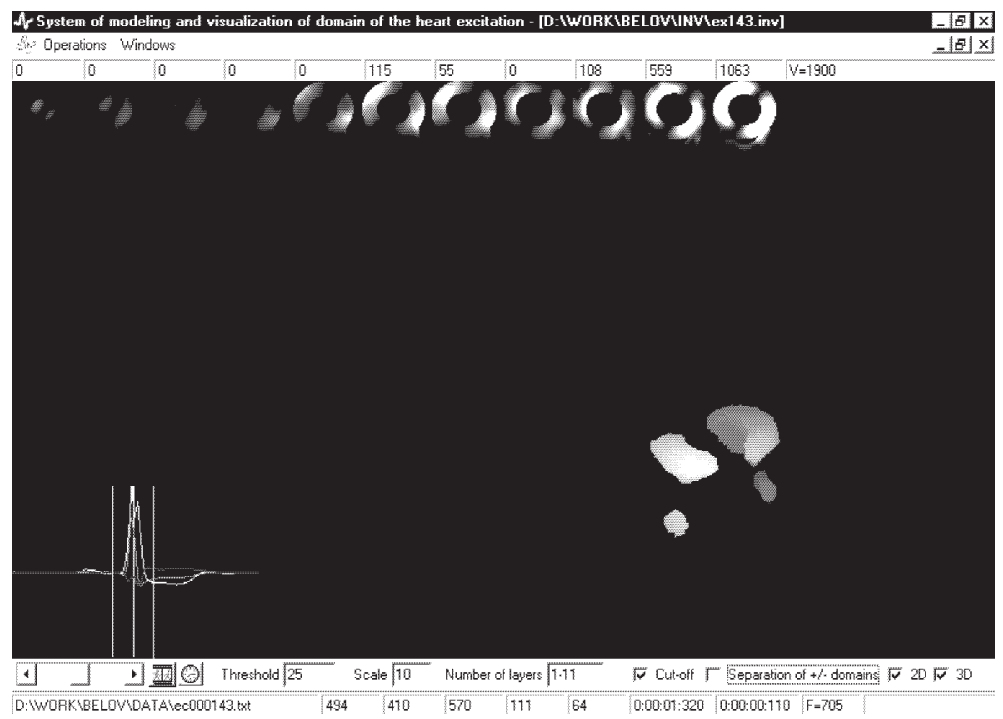

Fig.6. Excitation domain in ventricles zone for the sick man.

During of the test period (nine month) the 40 patients with myocardial infarction have been investigated. For all patients cardiologist determined correct diagnosis using our system. Thus developed system allows to determine visually part of the ventricles in which the break of the excitation spreading occurs. In 
addition cardiologist can estimate volume and distribution of the excitation domains. This results permit to use the system for the diagnostics and educational process.

\section{Future Goals}

The results of the paper can be developed to the following directions:

1. Use of the genetics algorithms solving $\Omega$-problem taking into account time segment restriction (6). This can permit to reconstruct desired domain more accuracy.

2. Advance of the system on the base of the non-truncation variants of the restrictions (4), (6). This can permit to diagnose cardiac irregularities more accuracy.

\section{Conclusions}

The new model of cardiogenerator is developed. This model operates within the framework of classical model of excitation spreading as a multidipole generator. At the same time our generator provides stable construction of domain of the heart excitation from the body surface potentials ( $\Omega$-problem) in contrast to multidipole one. The system of modeling and visualization of excitation process in ventricle of the heart is developed and clinical approved.

\section{References}

1. L. A. Lusternik, V. I. Sobolev. Elements of Functional Analysis. Moskwa, Nauka, 1965. 744

2. The Theoretical Basis of Electrocardiology. ed. by C. V. Nelson, D. B. Geselowitz. Clarendon Press, Oxford, 1976. 743, 745

3. L. I. Titomir. Electric Generator of the Heart. Moskwa, Nauka, 1980. 742, 743

4. A. Sezginer. The Inverse Source Problems of Magnetostatics and Electrostatics. Inverse Problems, 3:L87-L91, 1987. 742

5. D. B. Geselowitz. To Electrocardiogram Theory. IEEE Transactions, 77(6):34-55, 1989. 742

6. A. N. Tikhonov, A. V. Goncharsky, V. V. Stepanov, A. G. Yagola. Numerical Methods for the Solution of Ill-posed Problems. Moskwa, Nauka, 1990. 743, 745

7. F. Greensite. A New Method for Regularization of the Inverse Problem of Electrocardiography. Mathematical Biosciences, 111(1):131-154, 1992. 742

8. V. P. Ivanov, A. S. Batrakov. Three-Dimensional Computer Graphics. Moskwa, Radio i svjazj, 1995. 746

9. D. I. Belov, R. H. Sadykhov. Computer Simulation of the Inverse Problem of Electrocardiography. Proceedings of the 22 Annual International Computer Software and Applications Conference (IEEE COMPSAC'98), Vienna, 563-567, 1998. 744

10. D. I. Belov, R. H. Sadykhov. New Approach to Hidden-surface Elimination of 3D Medical Images. Machine Graphics and Vision, 7(4):831-840, 1998. 746 